\title{
Phenomenology and space-time picture of hadronization: past, present and future
}

\section{S.M. Eliseev*}

Bogoliubov Laboratory of Theoretical Physics

Joint Institute for Nuclear Research 141980 Dubna Russia

E-mail: selisetheor.jinr.ru

We start with a short review of the history of multiparticle production, Landau-PomeranchukMigdal effect (LPM), the new trend of this subject and its role in the explanation of some contemporary experimental data at high energy. We developed a cascade model of multi-production from Deeply Inelastic lepton-nucleus Scattering (DIS). On the phenomenological level, the model describes a Markov branching process of the evolution of parton showers (up to hadronization) in the atomic nuclei. The process of generation of particles is simulated by the Monte Carlo method. The formation time was extracted from comparison of the calculations and experiment on semi-inclusive DIS on nuclei.

XXII International Baldin Seminar on High Energy Physics Problems September 15-20, 2014

JINR, Dubna, Russia

* Speaker. 


\section{Introduction: a brief history of multiparticle production}

The problem of multiparticle production is one of the central problems in elementary particle physics and is connected with the problem of deconfined quarks and gluons (Quark Gluon Plasma, QGP) which is predicted by the quantum chromodynamics (QCD). Moreover, the hadron-hadron collisions are mainly inelastic, $\sigma_{e l} / \sigma_{i n} \lesssim 0.2$, and reflect the important fundamental property of

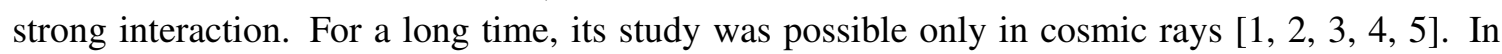
spite of great experimental difficulties, connected with considerable errors, cosmic ray physics laid down the foundations of our notions about multiparticle production. Nevertheless, a number of fundamental results where obtained in the experiments with cosmic rays. Scientific researches using cosmic rays in the 1930s and 1940s led to the discovery of particles such as the anti-electron or positron (first antiparticle); the muon (first second-generation matter particle); the pion (first meson); and both the kaon and the lambda (first particles containing the strange quark).

A few important peculiarities of multi-particle production were discovered in the experiments with cosmic rays: leading particle effect, concept of fireball and multiparticle collisions at high energy:

1.) In high energy hadronic collision only a small part of energy is spent for the production of particles. Inelasticity of hadronic interaction is defined as the fraction of the available energy go in for secondary particles. The remaining part of the incident energy $(\approx 60-70 \%)$ is carried away by leading particles.

2.) For the interpretation of jets from cosmic ray interactions, the group of physicists (see review of M. Miesowicz []]) proposed the mechanism of particle production. According to that mechanism, in the inelastic interaction two leading particles and one (or two) collision system (named fireball) have created. And the most of secondary particles are produced from the fireball. It was shown that the experimental data of cosmic rays interactions can be interpreted in framework of fireball model. Furthermore, the goal of current investigations in heavy-ion collisions is to establish the existence of the QGP and here is widely used concept of fireball, see Ref.[G] titled "Quark-Gluon Plasma Fireball".

3.) In particle-nucleus interaction at high energy, the particles produced in an interaction inside a nucleus are sufficiently collimated to interact simultaneously in the next collision inside the nucleus. In the extreme case, all the particles emitted from the first interaction simultaneously collide with the next nucleon. In a few collisions it is possible to double the energy available in the c.m. systems of successive collisions. (Cumulative effect? [四, []]). The idea of having the collisions pile up in the nucleus was published by M.F. Kaplon and D.M. Ritson in Ref. [四].

Modern accelerators have made possible the thorough and detailed investigations of multiparticle production in a large energy interval. Creating a fireballs of high density and high temperature, physicists are able to produce the particles that were copious in the early universe, immediately after the Big Bang. (On the largest accelerator in the world-the Large Hadron Collider at CERN.)

\section{The LPM effect: past, present and current trends}

Bremsstrahlung and pair creation are two of the most common high energy electromagnetic processes, and the interaction cross sections are well known. The spectrum of the bremsstrahlung 
of photon by a charged particle was described by H.A. Bethe and W. Heitlet [ $[\mathbf{\square}]$,

$$
d N \sim d k / k
$$

When an ultrarelativistic electron emits a low-energy photon via bremsstrahlung, the longitudinal momentum transfer between the electron and the target nucleus can be very small. Because of the uncertainty principle, this means that the momentum transfer must take place over a long distance, known as the formation length. One way to think of this is as the distance required for the electron and photon to separate enough to be considered separate particles. More than sixty years ago, Ter-Mikaelian introduced the concept of the so-called formation length for the emission of radiation [ [ $]$ ]. This is, just saying, the length it takes to separate the photon from the electron by one wavelength such that the photon can be considered as "formed". If the electron is influenced during this formation, the yield of photons may increase as when electrons traverse crystals or it may be reduced due to destructive interference. Landau and Pomeranchuk showed that if the formation length of bremsstrahlung becomes comparable to the distance over which the multiple scattering becomes important, the bremsstrahlung will be suppressed. Migdal developed the quantitative theory of this phenomenon. The formation length is the basic parameter of the LandauPomeranchuk-Migdal (LPM) effect [Q] ], which predicts a reduction of photon yield due to multiple scattering in the formation zone.

When a charged particle is moving in a medium it scatters on atoms. With probability $\sim \alpha$ this scattering is accompanied by a radiation. At high energy the radiation process occurs over a rather long distance, known as the formation length $l_{c}$ (see [ [8, Q Q]):

$$
l_{c}=\frac{l_{0}}{1+\gamma^{2} \vartheta_{c}^{2}}, \quad l_{0}=\frac{2 \varepsilon \varepsilon^{\prime}}{m^{2} \omega}
$$

where $\omega$ is the energy of emitted photon, $\varepsilon(m)$ is the energy (the mass) of a particle, $\varepsilon^{\prime}=\varepsilon-\omega$, $\vartheta_{c}$ is the characteristic angle of photon emission, the system $\hbar=c=1$ is used.

The LPM effect stems from the formation length, the distance over which an interaction such as pair production or bremsstrahlung occurs. For bremsstrahlung from an isolated atom, this distance is

$$
l_{f}=\frac{2 \hbar E(E-k)}{m^{2} k}
$$

where $E$ is the incoming electron energy, $k$ is the photon energy and $m$ the electron mass. This dis-

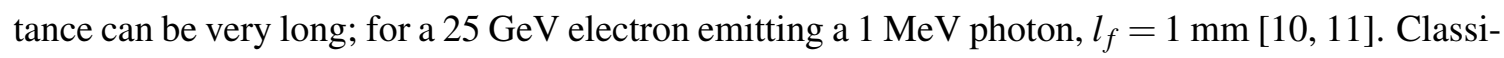
cally, if the electron is disturbed while traversing this distance, then the emission can be disturbed. In field theoretical language, other interactions within $l_{f}$ can interfere with the bremsstrahlung, reducing its amplitude. In dense media, the Bethe-Heitler, $1 / k$, bremsstrahlung photon spectrum is suppressed by a factor $S[\mathbb{W}]$ :

$$
S=\frac{\sigma}{\sigma_{B H}}=\sqrt{\frac{k E_{L P M}}{E(E-k)}}
$$

where $E_{L P M}=m^{4} X_{0} / E_{s}^{2}$ where $X_{0}$ is the radiation length of the material, and $E_{s}=m \sqrt{4 \pi / \alpha}$. The interactions of the electron throughout formation zone affect the radiation spectrum significant and may lead to enhancement (as in the case of crystals), or reduction of total intensity as well as changes in the spectral shape. 
The momentum transfer $q_{\|}^{\min }$ by electron of momentum $p$ and mass $m$ radiating a photon of momentum $k \equiv(1-x) p$, is defined by $q_{\|}^{\min }=m^{2}(1-x) / 2 p x$. From the uncertainty principle the formation length is given by $l_{f} \sim 1 / q_{\|}^{\min }$. At high energies, $p<<m$, and soft photon, $(1-x)<<$ $1, l_{f}$ can became large than the scattering men free path, $l_{f}>l_{m . f . p .}$, of electron. Landau and Pomeranchuk showed that the Bethe-Heitler spectrum is modified by the multiple scattering of the electron in dense matter. If the mean free path of the electron, $\alpha L$, is less than the formation length, $l_{f}$, the spectrum radiated photon is suppressed achieving the form $d N \sim d k / p \sqrt{L k}$.

Several experiments have presented evidence for the LPM effect. The SLAC experiment for the bremsstrahlung energy loss of 149,207 , and $287 \mathrm{GeV}$ electrons can be considered a truly successful systematic study of the effect in amorphous targets [ए, ए]]. This experimental study stimulated a quantity of theoretical interest. In Fig. 1 one result of this experiment for energiey 287 $\mathrm{GeV}$ in Ir is shown. A comparison between experimental values, the LPM suppressed spectrum and the unsuppressed Bethe-Heitler type spectrum clearly shows the strong suppression. As was shown in this experiment, the suppression increase with increasing beam energy.

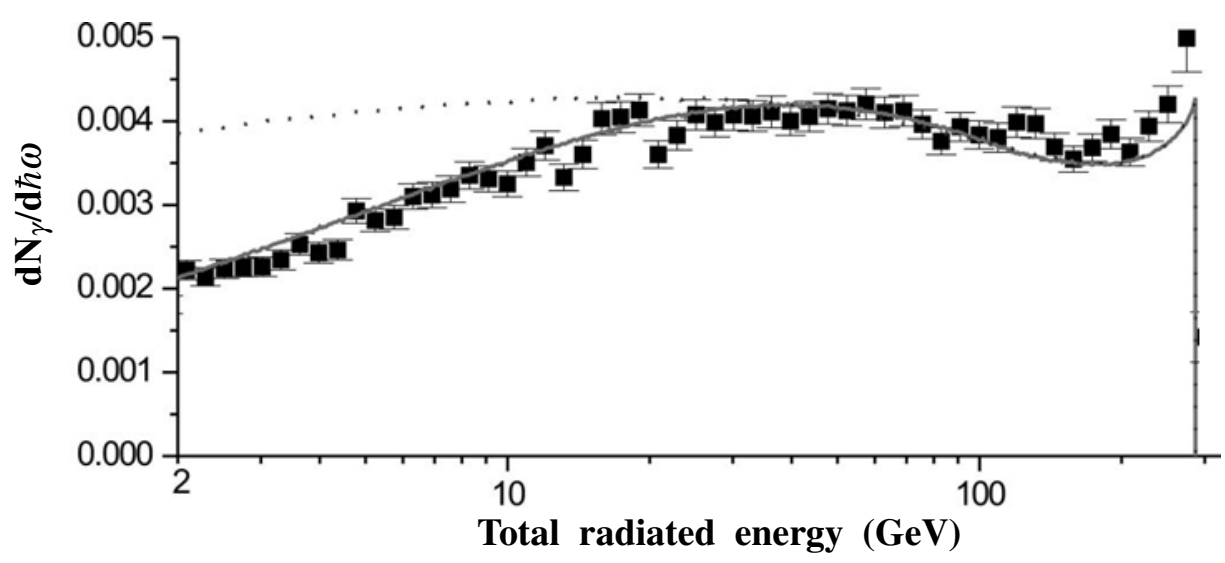

Figure 1: Bremsstrahlung spectrum, $d N_{\gamma} / d \hbar \omega$, for $287 \mathrm{GeV}$ electrons on $0.128 \mathrm{~mm}$ Ir. The total radiated energy, $\hbar \omega$, is presented in logarithmic bins ( 25 per decade) and plotted on a logarithmic scale. The vertical scale is normalized to the number of incoming electrons. The dotted line is the result of a simulation based on a pure Bethe-Heitler spectrum while the full line includes the LPM suppression [W]].

Originally, the LPM effect referred to the suppression of electron bremsstrahlung or pair production due to multiple scattering. The role of long distances in physics of strong interactionshadronic LPM effect-was noticed by Feinberg [ए2]. He showed that after the high energy scattering the electron "shakes off" its field and until this field is re-established the scattering takes placed without emission of a photon leading to suppression of the radiation.

The LPM effect must be taken into account in the study of many phenomena in high energy physics. It has a significant impact on the behavior of air showers in the neighborhood of the Greisen-Zatsepin-Kuzmin cutoff of high energy photons.The conflict between the existing data on cosmic rays and the GZK cutoff has led many theoretical workers to suggest that laws of physics must be modified [1[3], [4]].

More recently, it has been applied to the effects of the nuclear medium on quark and gluon interactions. This subject is of interest because calculations predict that quark or gluons should 
radiate increased energy in traversing a QGP, compared to normal nuclear matter. It has been established that the (LPM) effect is important in QCD although it was in the beginning found in QED. The LPM effect in QCD is especially valuable for estimating the energy loss, $\mathrm{dE} / \mathrm{dz}$, of an energetic parton traversing a dense QCD medium [15, [0]].

\section{The cascade model: Hadron formation in semi-inclusive DIS}

The interactions of ultrarelativistic heavy-ion collisions give the possibility to study many problems of QCD: the manifestations of quark and gluon degrees of freedom in nuclei, phase transition between ordinary matter and QGP, and so on.

At the same time, most of the experimental data in, e.g. RHIC's, have been of inclusive character, and some important information may be lost. A strict treatment of the results from RHIC is beyond our present theoretical possibility. A simplified models have been proposed to describe the mechanism of multi-particles production. These models describe the main points of macroscopic or microscopic peculiarities of the collision process. The intranuclear cascade (INC) model, together with its few variants, is example of microscopic approach. Also, INC calculations are free from adjustable phenomenological parameters. Because of these features, INC calculations are widely used for extracting of dynamical information connected with the mechanism of particles and nuclei interaction. INC can be used as an unbiased background for new phenomena [ㄱ] ]

The investigation of the quark propagation and hadron formation processes in a nuclear medium would greatly advantage the study of the QGP and its evolution in time. In addition, they may help to constrain the interpretation of the jet quenching seen in heavy ion collisions at the LHC and at RHIC.

Quark fragmentation has been intensively investigated in the past years by studying hadrons produced in lepton-nucleon scattering. The interactions of $v_{\mu}$ with the quasi-free proton and neutron in a deuterium target offer the opportunity of studying specific properties of a large variety of quark and diquark systems. According to the quark parton model, the dominant mechanism of charged current interactions consists of the interaction of a neutrino with a d valence quark which is converted into a u quark that subsequently is dressed up by quarks and antiquarks created in the colour field, to form a number of particles moving in the forward direction of the centre of mass system. The remaining diquark ( $u u$ for $v_{\mu} p$ and $u d$ for $v_{\mu} n$ ) yields backward going hadrons, Fig.2.
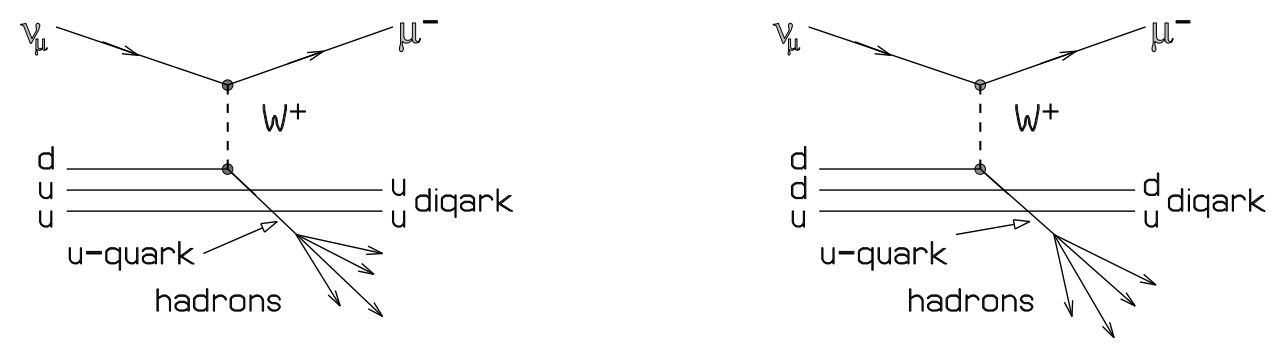

Figure 2: The dominant mechanisms for neutrino proton (Left panel) and neutrino neutron (Right panel) charged current scattering in the framework of the quark parton model.

In Deep Inelastic Scattering on nuclear targets one observes a suppression of hadron production analogous to hadron quenching in heavy-ion collision at the Relativistic Heavy-Ion Collider (RHIC). Moreover, the nucleons act as femtometer-scale detectors allowing to experimentally study 
the propagation of a parton in this "cold nuclear matter", and its space-time evolution into the observed hadron. In the case of heavy ion collisions, one wants to use hadron suppression as a tool to extract the properties of the hot and dense system created in the collision, also called "hot nuclear matter".

The conceptual ideas of our model are very straightforward and transparent. We assume that the interaction between incident an lepton and a target nucleus takes place in a lepton-nucleon interaction. The nucleus is excited by a series of collisions between secondaries (partons, produced in the first lepton-nucleon interaction) and the intranuclear nucleons. At high energies the secondaries traverse the nucleus in such a short time that nucleons cannot rearrange themselves until the probe has left. In the main the target nucleons are just static spectators, so that the scattering problem is in the first approximation a sequence of two-body ones. This process continues until all secondaries escape target nucleus. A part of the energy is spread through the nucleus to produce a fully-equilibrated nucleus which then decays statistically. The process of generation of particles is simulated by the Monte Carlo method.

The cross sections of intranuclear interactions and the characteristics of secondary particles (multiplicities, rapidity and transverse momentum distributions) at different energies are taken from experiments with free nucleons [ㅍ]].

Elastic and inelastic interactions, charge exchange and absorption of slow pions inside the nucleus are taken into account. Nuclear effects such as the Fermi motion and Pauli blocking are all taken into consideration.

Since the interaction cross section of neutrino with a nucleon is small, we assume that the neutrino can interact with any nucleon of the nucleus with equal probability, i.e.

$$
\sigma(v+A)=\sum_{i=1}^{A} \sigma_{i}(v+N)
$$

where $\sigma_{i}(v+N)$ is the interaction cross section of a neutrino with a separate nucleon. Following experiments and the quark parton model, we take into account that the interaction cross section of neutrino with a neutron is twice that with a proton, i.e. $\sigma(v+n) / \sigma(v+p) \approx 2$.

The Fermi gas model of the nucleus is utilised. The nuclear density is taken from the WoodsSaxon expression

$$
\rho(r) \sim 1 /\left(1+e^{(r-c) / \alpha}\right)
$$

with the parameters $\alpha=0.57 \mathrm{fm}$ and $c=1.19 A^{1 / 3}-1.61 A^{-1 / 3} \mathrm{fm}$. Moreover, three-dimensional geometry is used in our calculations.

Then, knowing the nuclear density, we can place A nucleons inside the nucleus. For each simulation of A nucleons, we fix the coordinates of the center of intranuclear nucleons throughout the process of developing the cascade. The characteristics of charged particles produced in the first $v \mathrm{~N}$ interaction inside the nucleus, are taken from experimental data, even - and odd - prong events, see Fig.3. (The parton spectra is assumed to be the same as hadronic one. That approach is based on the concept of "Local Parton Hadron Duality".)

The multiplicities of neutral particles (pions) are taken from the experiment. These multiplicities for $v \mathrm{n}$ and $v \mathrm{p}$ are respectively

$$
n_{\pi^{0}}=0.72+0.22 n_{\pi^{-}}
$$


and

$$
n_{\pi^{0}}=0.14+0.73 n_{\pi^{-}}
$$

where $n_{\pi^{-}}$is the multiplicity of negatively charged particles. The angular and momentum distributions of neutral particles are assumed to be the same as those of pasitively and negatively charged particles. The space-time characteristics of lepton-nucleon interactions inside the target nucleus were taken into consideration. We use the concept of so-called the time - dependent cross section introduced in Ref. [19]. The hadron formation time, $\tau$, is the time between the moment that the parton is produced and the moment that the final pre - hadron is formed. After time $\tau$ from the intranuclear collision, the time - dependent cross section for the next colission of a secondary particle with a nucleon inside the nucleus is given by [ए]]

$$
\sigma_{h N}=\sigma_{h N}^{\exp }\left(1-e^{-\tau / \tau_{f}}\right)
$$

where $\sigma_{h N}^{e x p}$ is the experimentally determined interaction cross section of a hadron with a free nucleon. Thus, only after a relatively long time $\tau$ does the cross section of intranuclear interaction reach the value $\sigma_{h N I}^{\text {exp }} . \tau_{f}$ is the parameter of formation time.

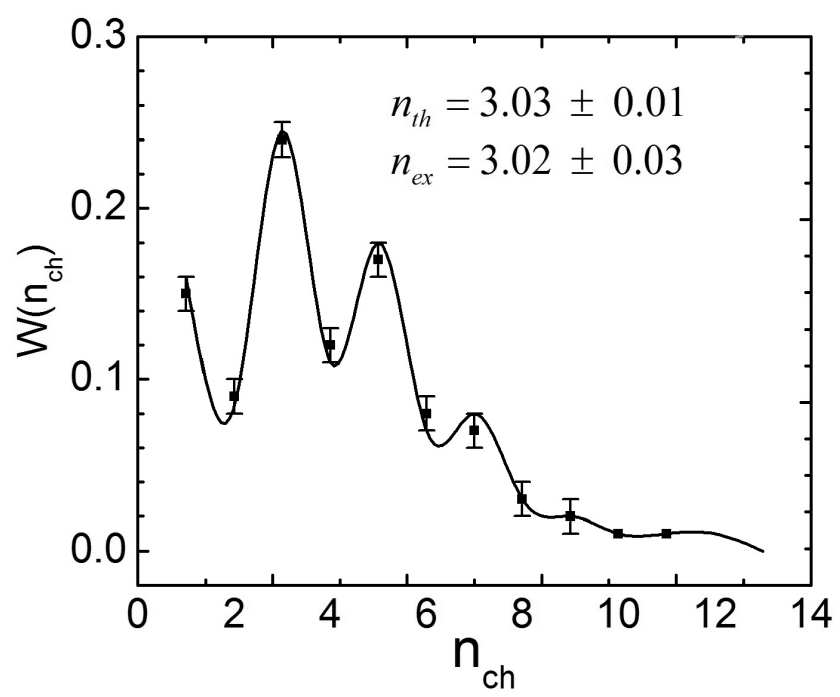

Figure 3: The multiplicity distribution of particles for charged-current $v_{\mu}$-d interactions in nuclei. $\left(v_{\mu}+p \rightarrow \mu^{-}+X^{++}, X^{++} \rightarrow\right.$ hadrons, even-prong events; $v_{\mu}+n \rightarrow \mu^{-}+X^{+}, X^{+} \rightarrow$ hadrons, odd-prong events.) The curve corresponds to the distribution of partons (in the framework of the LPHD concept) used in our model. The experimental data are taken from Ref. [ए8].

Below, we present the theoretical results at different zone formation, $l_{f}$, in the system of moving particle for neutrino $-{ }^{20} \mathrm{Ne}$ interaction and compare them with experimental data.

A comparison between theoretical calculations and the experimental data is presented in Table 1, which clearly shows that:

1. The multiplicities of particles from $v_{\mu}-{ }^{20} \mathrm{Ne}$ interactions (calculated on our model) depend on zone formation $l_{f}$.

2. The model based on simple rescattering $\left(l_{f}=0\right)$ can't describe particle production.

3. Zone formation of pions, $l_{f}(\pi) \approx 0.5 \mathrm{fm}$. 
Table 1: The multiplicities of charged hadrons produced in charge-current neutrino $-{ }^{20} \mathrm{Ne}$ interaction: the theory vs. experimental data.

$\left.{ }^{*}\right)$ for $\pi^{ \pm}$mesons and protons with $p_{p} \geqq 1 \mathrm{GeV} / \mathrm{c}$,

(**) for protons with $0.35 \leqq p_{p} \leqq 0.8 \mathrm{GeV} / \mathrm{c}$. The experimental data are taken from Ref.[ए8]].

\begin{tabular}{|c|c|c|c|c|c|c|}
\hline \hline Particles & Experiment & Experiment & \multicolumn{4}{|c|}{ Calculations on our model } \\
\cline { 4 - 7 } & E172 & E546 & $l_{f}=0$ & $l_{f}=0.5 \mathrm{fm}$ & $l_{f}=0.2 \mathrm{fm}$ & $l_{f}=0.1 \mathrm{fm}$ \\
\hline$<n\left(\pi^{-}\right)>$ & $1.37 \pm 0.04$ & $1.36 \pm 0.03$ & $1.54 \pm 0.01$ & $1.41 \pm 0.01$ & $1.33 \pm 0.01$ & $1.27 \pm 0.01$ \\
\hline$<n^{ \pm}>^{*}$ & $3.98 \pm 0.11$ & $4.20 \pm 0.06$ & $4.70 \pm 0.02$ & $4.20 \pm 0.02$ & $3.81 \pm 0.02$ & $3.65 \pm 0.02$ \\
\hline$<n_{p}>^{* *}$ & & $0.33 \pm 0.02$ & $0.32 \pm 0.01$ & $0.27 \pm 0.01$ & $0.22 \pm 0.01$ & $0.12 \pm 0.01$ \\
\hline \hline
\end{tabular}

4. Zone formation of protons, $l_{f}(p) \ll l_{f}(\pi)$. This is in the consistent with the QPM in which it is assumed a difference in the fragmentation of quarks and diquarks. (Diquarks $u d$ is produced from the interaction of $v_{\mu}$ with neutron of nucleus, proton is formed when diquark $u d$ picks up $u$ quark from the vacuum, see Fig. 2).

\section{Summary and outlook}

In summary, the parton propagation in nuclear matter and hadron production in neutrino $-{ }^{20} \mathrm{Ne}$ deep inelastic scattering is studied in our model by using the concept of time - dependent cross section (see Eq. (3.5)). The results of the calculations are compared with the experimental data and the distance of parton hadronisation, $l_{f}$, in nucleus is determind. (It worth noticing that our results have been obtained without fitting any new parameters.) At present, the semi-inclusive deep inelastic scattering of lepton on nuclear target has been one of the most active frontiers in nuclear physics and particle physics. Newly data have been obtained from the HERMES experiment at DESY and from CLAS collaboration at Jefferson Lab.

Using the cold nuclei, the time development of hadronisation processes is studied in the well known nuclear system as a spatial analyzer. In the case of relativistic heavy ion collisions, the same processes can be used as tools to explore the nature of the hot dense matter. There have already been some investigations using time-dependent cross sections for the description of final state interactions in ultra-relativistic heavy-ion collisions [[0]].

\section{References}

[1] W. Heisenberg, Z. Phys. 101, 35 (1936); Z. Phys. 101, 533 (1936).

[2] W. Heisenberg, Kosmische Strahlung, Berlin, Springer - Verlag, 1943.

[3] "Some remarks about fireballs,"

M. Miesowicz, Acta Phys. Polon. A 38495 (1970) and references therein.

[4] "Emulsion Cloud-Chamber Observations on the Interactions of High Energy Primary Cosmic Radiation," M.F. Kaplon and D. M. Ritson, Phys. Rev. 110, 368 (1952).

[5] "Intranuclear cascades with many-particle interactions,"

I.Z. Artykov, V.S. Barashenkov, S.M. Eliseev, Nucl. Phys. B 6, 11 (1968). 
[6] "Quark-Gluon Plasma Fireball,"

Salah Hamieh, Jean Letessier and Johann Rafelski, Phys. Rev. C 62, 064901 (2000).

[7] H.A. Bethe, and W. Heitler, Proc. Roy. Soc.,A 146, 834 (1934).

[8] M.L. Ter-Mikaelian, Zh. Eksp. Teor. Fiz.25, 289 (1953).

[9] L.D. Landau and I.J. Pomeranchuk, Dokl. Akad. Nauk. SSSR 92, 535 (1953);

A.B. Migdal, Phys. Rev. 103, 1811 (1956);

Pomeranchuk, I.J. and Feinberg, E.L., Dokl. Acad. Nauk SSSR, 93, 439 (1954). 1811 (1956).

[10] H. D. Hansen et al., Phys. Rev. Lett. 91, 014801 (2003);

R. Bellwied1, C. Markert, Phys.Lett. B 691, 208 (2010).

[11] H. D. Hansen et al., Phys. Rev. D 69, 032001 (2004) and referenses therein.

[12] E.L. Feinberg, in Problems of theoretical physics, Moscow: Science, 1972, pp. 248.

[13] "Ultra-High Energy Quenching of the LPM Effect: Implications for GZK-Violating Events," John P. Ralston et al, arXiv:0209455v1 [astro:ph].

[14] Lisa Gerhardt and Spencer R. Klein, Phys.Rev. D 82,074017 (2010).

[15] "The Ter-Mikayelian Effect on QCD Radiative Energy Loss", Magdalena Djordjevic and Miklos Gyulassy, Phys.Rev. C 68034914 (2003).

[16] "LPM effect as the origin of the jet fragmentation scaling in heavy ion collisions", Frashër Loshaj and Dmitri E. Kharzeev, Int. J. Mod. Phys. E 21, 1250088, (2012).

[17] T. Kodama et al., Phys. Rev. C 292146 (1984);

G.Bertsch et al., Phys. Rev. D 37, 1202 (1988);

T.J. Humanic, Nucl.Phys. A 715 (2003), 0205053 (2003);

T.J. Humanic, Acta Phys. Hung. A 25, 0203004 (2006).

[18] J.W. Chapman et al., Phys. Rev. Lett., 36, 124 (1976);

W.M. Yeager et al., Phys. Rev.D 16,1294,(1977); T.H. Durnett et al., Phys. Lett., B 77, 443 (1978);

M. Derrick et al., Phys. Rev. D 17, 1, (1978) and referenses therein.

[19] A. El-Naghy and S.M. Eliseev, J. Phys. G: Nucl. Part. Phys. 16, 39 (1990); M. J. van Goethem et al., Phys. Rev. Lett. 88, 122302 (2002);

K. Gallmeister, U. Mosel, Nucl. Phys. A 80168(2008).

[20] K.Gallmeister, C. Greiner and Z. Xu, Phys. Rev. C 67, 044905 (2003);

W. Cassing, K. Gallmeister and C. Greiner, Nucl. Phys. A bf 735, 277 (2004);

K. Gallmeister and U. Mosel, Nucl. Phys. A 801, 682008. 